\title{
Sensory Probes: An Exploratory Design Research Method for Human-Food Interaction
}

Tom Gayler*, Corina Sas*, Vaiva Kalnikaitè ${ }^{\wedge}$

*School of Computing and Communications, Lancaster University, United Kingdom

^Dovetailed Ltd., Cambridge, United Kingdom

Designing interactions with food holds potential for rich multisensory experiences but their pervasiveness can challenge our understanding of them. This paper presents the design and evaluation of Sensory probes, a novel, exploratory design research method aimed to sensitize participants towards their food experiences. We report on workshops with 8 participants for co-designing the probes, followed by iterative revision through two-week diary studies with 18 participants. Findings indicate strong engagement with the sensory probes and how they brought forward the bodily and sensory aspects of these experiences, alongside emotional and social ones. We highlight the design rationale for the sensory probes which has been both empirically- and theoretically-grounded, provide reflections on the value of these probes for enabling novel perspectives on food experiences, and on probes' ability to capture what we called sensory fragments of participants' experience reflecting distinct sensory aspects form both internal and external senses.

CCS CONCEPTS $•$ Human-centered computing $\sim$ Interaction design $\sim$ Interaction design process and methods $\sim$ User centered design $\bullet$ Human-centered computing $\sim$ Interaction design $\sim$ Interaction design theory, concepts and paradigms

Additional Keywords and Phrases: Probes, Multisensory Design, Exploratory Design Research Methods, Food, Flavor, Experience

\section{Introduction}

Food experiences are multisensorial, embodied, emotional, social, and cultural offering rich design opportunities. These qualities have fostered the growing HCI interest in human-food interaction (HFI) over the last decade, while contributing also to the HCI agenda of multisensory research. Such work has led to innovative technologies for, and around food experiences, from 3D food printers to virtual reality systems. However, design methods supporting the exploration of this fecund design space have received limited attention. Building on the multisensory richness of food experiences, we argue for the value of injecting sensory qualities to the traditional cultural probes as novel exploratory design research methods for human-food interaction. While often probes are presented as end products, in this paper we take a different approach unpacking the iterative process of creating and evaluating them through a co-design process involving workshops for designing the probes with 8 participants, followed by iterative revision through two two-week diary studies with 18 participants in total. We introduce the sensory probes (Figure 1), a novel exploratory design research method consisting of five artifacts, namely a 'TasteWho' gameboard pairing flavor descriptors with bespoke evocative images; letters with prompts for writing meaningful recipes; eye mask, nose clip, ear plugs and gloves for sensory augmentation; body mapping booklet; and camera and sound recorder for capturing environmental context. Our work aims to address the following research questions:

- How can we co-create a package of sensory probes to support the design of personalized food experiences?

- What new affordances and roles such do sensory probes have? 
- How do sensory probes contribute to the design research for multisensory experiences in general, and food experiences in particular?

Our contribution is threefold. First, we introduce the Sensory probes, a novel exploratory design research method; second, we provided both empirically- and theoretically-grounded rationale for the design of sensory probes; and finally, we reflect on their value for enabling novel perspectives on food experiences, and affordances for capturing sensory fragments, while engaging both interoceptive senses and external senses more broadly.
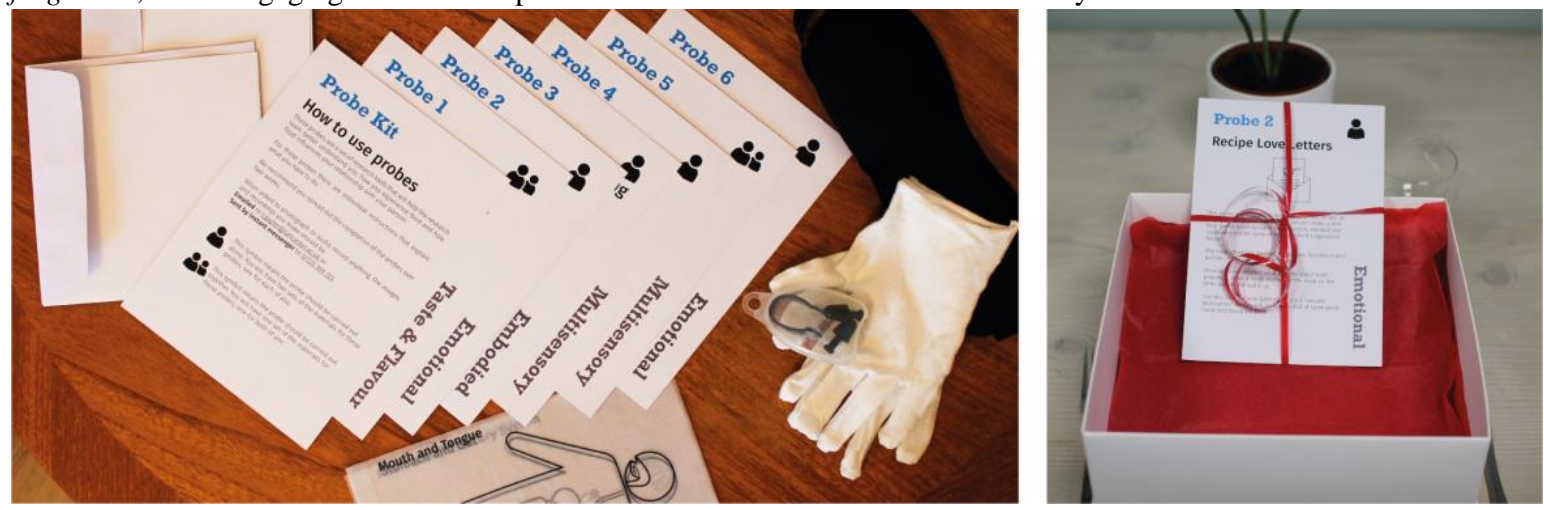

Figure 1 Probe kit elements (left) and probe kit packaged as gift (right)

\section{Related Work}

Our work draws from three HCI research areas that have developed largely independently, with limited integration, such as work on designing for human-food interaction [23], multisensory interaction $[14, \underline{42}, \underline{56}]$, and design probes inspired by cultural probes [18].

\subsection{Designing for Human-Food Interaction}

The growing HCI interest in human-food interaction has explored our technologically mediated relationship with food in a variety of contexts [3]. This work has used technology to improve the experience of eating food through systems that promote healthier eating $[\underline{5}, \underline{26}, \underline{31}, \underline{38}]$, those employing augmenting sensory experience to improve liking of flavors $[\underline{34}, \underline{37}]$ to those supporting social interactions around dining $[\underline{35}, \underline{64}]$. Other strands of work have focused on using food to create new experiences and practices, $[\underline{20}-\underline{22}, \underline{29}]$, supporting play $[\underline{44}, \underline{61}]$, communicate data $[\underline{33}, \underline{49}]$, or explore scientific concepts through embodied experience [15, $\underline{36}$ ]. Despite its breadth, [24], most such work has employed traditional research and design methods, with limited effort towards novel exploratory design research methods tailored to the unique qualities of food as resource for design. The few attempts in this direction include a framework for designing temporal, embodied and affective experiences with taste [46], material food probes for the exploration of flavors in intimate relationships [23], drawing on 'play potentials' [2] to reinterpret existing cultural food experiences, and experimental food crafting to explore its value for new interactive food technologies [16]. These valuable contributions however are narrowly focused either on a single aspect of sensory experience (taste) or tied to a particular food material (liquid-based 3D printed food). In addition, the current exploration in human-food interaction has touched upon the different facets of food experiences such as personal, social, and cultural, albeit with a limited focus on the multisensory quality of food experiences. For this, we turned our attention to the broader HCI research in multisensory interaction, and its design methods.

\subsection{Multisensory Experiences in $\mathrm{HCl}$}

The advent of novel technologies supporting interaction through touch, taste, and smell has paved the way to new guidelines for multisensory design $[\underline{14}, \underline{42}, \underline{56}]$. Most such guidelines have framed multisensory experience as a 
compound experience that can be deconstructed into individual sensory ones through reference to body parts [56] or metaphors pertaining to synesthesia [42], which then can be used to reconstruct these individual sensory phenomena into broader multisensory experiences [14].

\subsubsection{Exploratory Design Research Methods: Cultural Probes as Multisensory Tools.}

Cultural probes are a much explored design research method [53]. From Gaver and colleagues' [18, $\underline{19]}$ seminal work introducing them as inspirational design research methods intended to provoke, humanize, use uncertainty and create fragments, probes have inspired a rich set of interpretations [25]. In the original form, cultural probes included a range of artifacts such as [18]: (i) postcards with prompting questions, (ii) maps to be annotated with participants' attitudes towards the researched topic, (iii) a camera with suggestions for what to photograph, (iv) a photo album to be curated by participants, (v) a media diary to capture use of TV and radio.

Gaver's cultural probes were not used to inform co-design but to support ongoing conversations with participants as they capture their world as it is. Probes also included tasks involving "ambiguity, absurdity, and mystery" [18] aimed to provoke alternative perspectives to participants' habitual experiences. Cultural probes aim to capture inspirational data concerning participants' beliefs and desires, rather than needs. The artifacts provided in the original package however tend to engage mostly with visual and auditory experience, with limited consideration of participants' multisensory experience and their body [을.

We argue that there is an opportunity to uncover also inspirational data about participant's sensory experience through probes [23] . For this, we can draw for instance from sensory ethnography [50] in order to fold in ways of knowing derived from sensory bodily experience. Doing so would make participants' bodies new sites of design interest, rather than for instance their local environment and communities [18]. Despite the rich variants of cultural probes, the focus has been mostly on how participants' gathered data sensitize and inspire designers, rather than on the design rationale for putting together the specific package of artifacts $[\underline{7}, \underline{62}]$.

\subsubsection{Bodily-Centered Design Approaches.}

An important aspect of designing for bodily and multisensory experiences is the ability to articulate idiosyncratic personal experiences [14]. Although co-designing approaches offer accessible ways to access them [9], novice users may find it challenging to become aware of, and articulate their embodied and sensorially rich experiences. While suggestions have been made for integrating sensory ethnography approaches into HCI design and research methods to better support design for and with the body [50], there has been limited progress in this direction to date.

Another strand of design methods includes bodily-based design approaches which have been purposefully tailored to support the articulation of bodily experiences. Body as a resource for design has received a growing HCI interest, both the body of participants $[\underline{12}, \underline{45}, \underline{51}, \underline{54}]$ and of designers $[\underline{1}, \underline{55}, \underline{58}]$. Grounded in phenomenology, the somaesthetics approach focuses on somatic experiences and our awareness of them, particularly while interacting with technologies. For instance, Aesthetic Laborations (A-labs) is a sensitizing approach which treats cognitive and bodily experience as entwined $[\underline{1}, \underline{28}]$. This approach augments sensory information through blindfolding users in order to explore materials through different senses and body parts, for example through touching one's back of hand or lips. Another bodily-based design approach is 'sensory bodystorming' [59], in which attention is paid to each sensory pathway in order to support ideation. The intention of such approaches is to better understand sensory and bodily experience with the aim of informing the design of novel multisensory interactions. They also aim to support awareness, understanding, and communication about personal sensory experiences that may be otherwise unnoticed. Another useful approach to this issue is Wilde and colleagues' [65] framework for embodied ideation based on 'disrupt-destabilize - emerge - embody' which aims to support expression of rich bodily experiences and to create estrangement or unfamiliarity to inform design. The framework proposes 4 methods to support new perspectives such as: re-contextualizing objects in new contexts, changing bodily sensations through artefacts and manipulation of sensory experiences; enacting instructed actions, and changing materials so they are used in different ways.

To conclude, with the emergence of body-centric methods in HCI, and the growth of HFI research, we argue that there is value in bringing together these rather separate strands in order to integrate food as a resource for designing with and for the body. Cultural probes offer a promising starting point to explore the breath of individuals experience with, and around food, as well as the to connect multisensory flavor experiences with social and cultural practices. This paper reports on the 
development of what we call sensory probes which purposefully extend cultural probes in order to better understand the sensory and bodily experiences with food.
8 participants
10 participants
8 participants

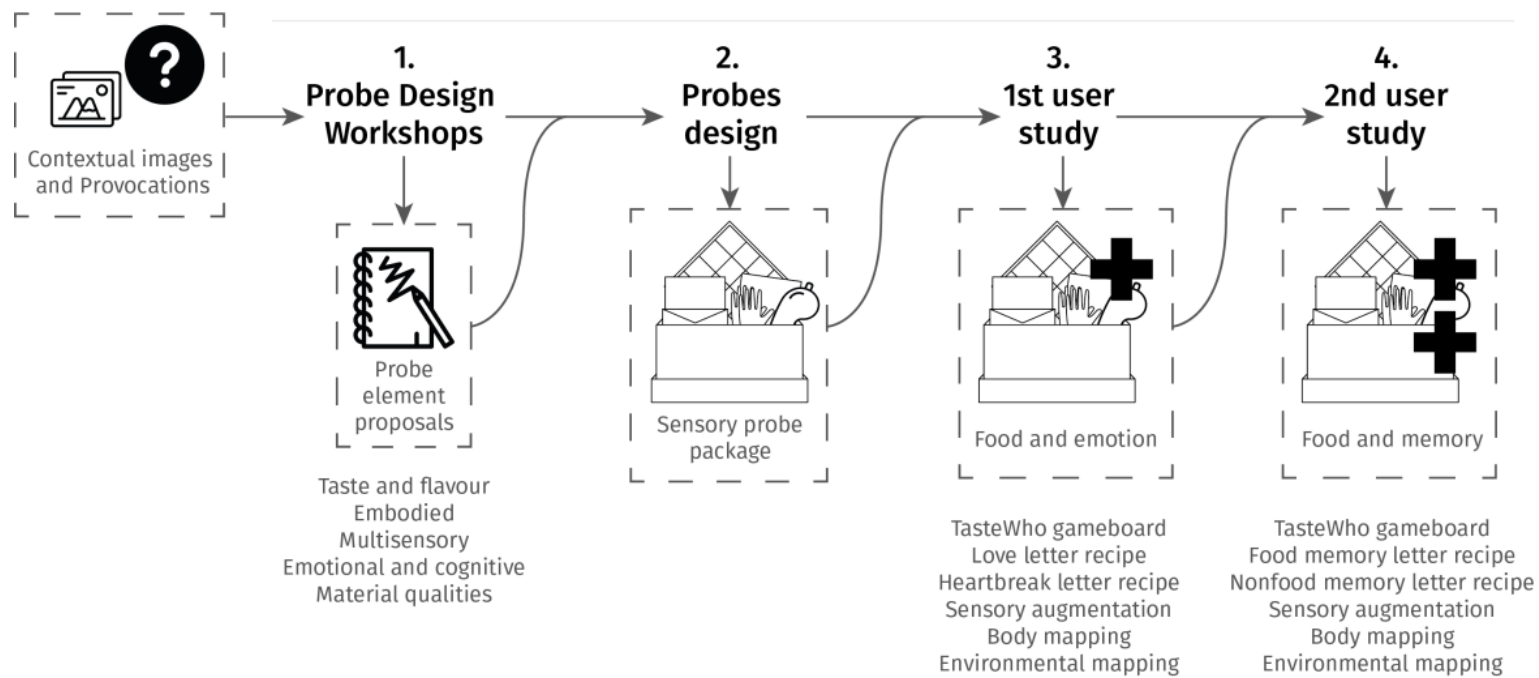

Figure 2 Method diagram for probe development

\section{Overview of Studies for Design \& Evaluation of Sensory Probes}

The sensory probes were developed through an iterative process starting with probes' design workshops with 8 participants (5 designers and 3 HCI researchers) (1, Figure 2). From these workshops, the first version of the sensory probes was designed and produced (2, Figure 2$)$ to be used and evaluated in a food-emotion study (3, Figure 2$)$ and a food-memory study (4, Figure 2$)$.

\subsection{Probe Design Workshops: Method}

We conducted two workshops with 8 participants in total: one workshop with 3 HCI researchers, (P1-3), and another one with 5 Design researchers, (P4-8). These were facilitated through cards with contextual images and sensitizing questions together with brief video/audio clips. Both workshops lasted 2.5 hours and were structured in the following 7 parts:

1. Introduction section described the aim of the workshops, namely, to gather insights into probes design and what artifacts are to be included in the package. Participants were introduced to the concept of probes inspired by $[\underline{7}, \underline{18}, \underline{19}]$, and were informed that the probe package will be used by participants in two follow up studies on food-emotions, and food-memories.

2. Building on the established linked between tastes and emotions [10], the taste and flavor section focused on participants' most memorable tastes and flavors.

3. Embodied experiences part aimed to sensitize participants to their body and bodily experiences. Here we asked questions such as: "What parts of the body interact with food?" "What reactions do our bodies have to food?" "How do we experience our intimate relationships through our bodies?"

4. Multisensory experiences section included sensitizing questions such as "How does smell/touch/sound/sight relate to the experience of food?", "What other senses should be considered in the probe design?"

5. Emotional and cognitive aspects of food experiences section focused on how these could be captured. 
6. Material qualities of food experiences part focused on questions such as "How does the physical state of food impact experience, i.e., solid, liquid, creamy, oily?".

7. In this final section, participants were asked to suggest different probes. Each participant generates several responses that were drawn, written, and verbally described so that they were shared and iterated in the group. The workshops generated hand-drawn and annotated sketches for various probes and their artifacts, alongside researcher's notes. These were analyzed by the first and second authors through an iterative process in order to identify recurring themes and to inspire the design of probes. While some probes drew directly from suggestions in the workshops, others were inspired by fragments of experience recounted. Selections of the final probes were made to generate a variety of experiences with food, both mundane and less familiar ones while leveraging rich experiences elicited or captured through multiple modalities including eating, writing, drawing, photographing and audio recording. This list was then prototyped and iterated between the team resulting in the first package of probes.

\subsection{Findings from the Probes Design Workshops}

We now present the main groups of food experiences that the participants reported as inspirational activities around the novel probes that we aimed to co-design with them. The probes were designed to complement one another, each covering one or more of the aspects of food experience below. Although potentially used individually to explore specific aspects of experience, the intention was that they should be used together to generate a richer, multi-dimensional perspective through combining insights from multiple probes.

\subsubsection{Embodied food experiences.}

All participants highlighted the value of the body for designing the probes. P2 and P5 drew diagrams of the body to highlight the role different parts played in food experience: "Tooth-temperature; tongue - texture, stomach-liquidity, anus - spicy" (P2), or "brain - freeze when eating ice cream ...stomach, normally feel quite heavy after eating/drinking especially during stressful period ... cheek gets sore after chewing hard things for a long time... finger - thumb and index finger get sore after picking things up a lot" (P4). This led to a group's proposal for considering how body parts might be used through probes for different aspects of food experiences and discussion of the stomach as a mirror of emotions. Both P1 and P4 considered the role of thermal experience of food and its emotional qualities: "cup of tea/coffee/cocoa; holding it with both hands to warm them very comforting as if someone was holding them too" (P1). Similar proposals were for probes that could provide indicators of fullness (P7) as expressions of bodily sensation. Alongside the thermal properties of the food, the way the food was eaten was also suggested as key for exploring embodied experiences: "food eaten from the finger tastes better" (P4), and "drinking wine from Porron de vino you have to hold it up maintain balance and point to mouth" (P1). As a result, the Body mapping probe took shape. These findings also informed the Sensory augmentation probe in which different objects are used to shape and emphasize different angles of the experience with food.

\subsubsection{Material qualities of food as probes.}

Also related to food experiences was the form factor of the food itself. For instance, P1 suggested using foods that can be customized by participants to allow them to express their own preferences and personalities through what they consume. Most participants were also in agreement that quantities of food to be consumed as part of interacting with probes should be "one mouthful with the possibility to have more" (P6) supporting the experience of "biting it" (P4). Participants also discussed if foods should be included that they would need a long-shelf life, perhaps limiting the range of potential experiences. These points informed how much food was to be consumed in the design of TasteWho gameboard, where they were kept small but enough for a bite, and the larger meals eaten with the Sensory augmentation and Body mapping exercises where the duration of experience with food was more important.

\subsubsection{Meaningful food experiences.}

Meaningful food experiences led to probes' concepts such as TasteWho game, Letters, or Diaries. One key aspect of emotional and cognitive experiences that participants thought that the probes should focus on was the role of favorite recipes. P5 saw "recipes [as a] performative" and proposed a probe artifact around "learning the dish of another person, learning their comfort food", an idea also proposed by P4. During group discussion this was extended to consider how 
recipes allow sharing and engaging in family history, as illustrated by the following quote: "being away from home, food brings me back and makes me feel closer to my family when I cook my grandma's recipes" (P1). The focus on emotional experiences also led to suggestions of probes that can allow the identification of "sad food vs happy food" (P6), something also mentioned by P4 and P8. Proposals from the groups also included food gifts, relationship recipes and "the most disgusting food challenge". The most disgusting food challenge concept related to the idea of a heartbreak letter and its associated food recipe. The process of creating such letter and recipe could be seen as a performative act that connected food with strong meanings. The acknowledgement of the importance of happy and sad foods also supported the diary concept for capturing the place where food was eaten and the associated mood. Emotional foods also led to the concept of TasteWho gameboard for selecting foods that matched specific emotions. This game idea highlighted novel relations between food and emotions where emotions can be both cause and outcome of consuming food.

\subsubsection{Identity food experiences.}

Identity was another cluster of food experiences, related to taste and preference, as noted by P4: "[tastes represent] growing up, cultural tastes, class". P2 similarly noted spicy and umami as "life-long important flavors". The value of tastes and flavor to support narratives that describe aspects of selfhood were also interlinked to important memories: "Lanzhou beef noodle soup, a regular food originated in Xinjiang [...] and every city in China has a slightly different taste to it [...] It's a cheap one that fills you on-the-go. My dad used to take me for it just around the corner. My friends and I went there a lot for lunch in high school you can smell the soup base far away, faintly but you can tell" (P5). This repeated episode connects family relations, friends, national identity, and sensory experiences, indicating that probes can be used to prompt explorations of self-identities. The potential for identity to be linked to ingredients or dishes was leveraged in the design of the Letters probe. Identity was most apparent in the letters used for the food-memory study in which participants were instructed to connect a specific recipe with an important memory.

\subsubsection{Playful food experiences.}

Another group of food experience were playful ones. Games related to taste and flavor were noted as potentially useful, in particular by P1, who describes "Wasabi-laced corn chips" and "very sour sweets I used to eat as a teenager; [we would] compete to see who could eat the most without a tear". Such experiences of play mediated through taste and flavor were mentioned by 4 participants. Key for play is challenging taste thresholds (such as very sour), strong, acquired tastes such as wasabi, and surprising combinations such as wasabi and corn. Playfulness also engages a social dimension, for instance the social experience of watching one's friends' "faces when eating [very sour sweets] without realizing, was priceless" (P1). Strong taste and flavor experiences also become memorable as P6 vividly describes the "sour [taste of] rotten tea, a green tea that has turned bad after 3 days. I have never forgotten memories of this from my childhood". Play in the form a game was influential in the development of the TasteWho gameboard, in particular the guessing of food as suggested by P1. This discovery of an unknown food was also used in the Sensory augmentation probe where the experience was altered, to generate uncertainty about the food being eaten.

\subsubsection{Contextual food experiences.}

When considering how multisensory experiences could be explored, participants also mentioned environmental factors and how changes in sensory capability affect perception. P8 listed a series of aspects they felt impacted on the experience, related to both sensory factors: "lighting, position, warm/cold, noise, seating - hard/soft, supported", while P4 noted factors such as the "heat of the room helps with comfort; or smell of cake cooking" so these as well may be considered by probes. Sounds were also identified as influential for designing probes, as P5 speculated how we design multisensory spaces with "light and sound, candles [to create] romantic atmosphere". As well as constructing sensory environments, participants also considered how restricting some sensory experience might increase awareness of others, as "eating without seeing [you] focus all your senses on the food" (P6). Consideration was also given to capturing the context of the food experiences within the probes, and P5 argued that participants should "document what environment, food was chosen to be eaten in". For capturing these contextual aspects of experience, participants referred to traditional audio recording or photo taking, with social aspects of food experiences being also captured through diaries. These contextual aspects inspired the Sensory augmentation probes and the TasteWho gameboard with its social, intimate context. 


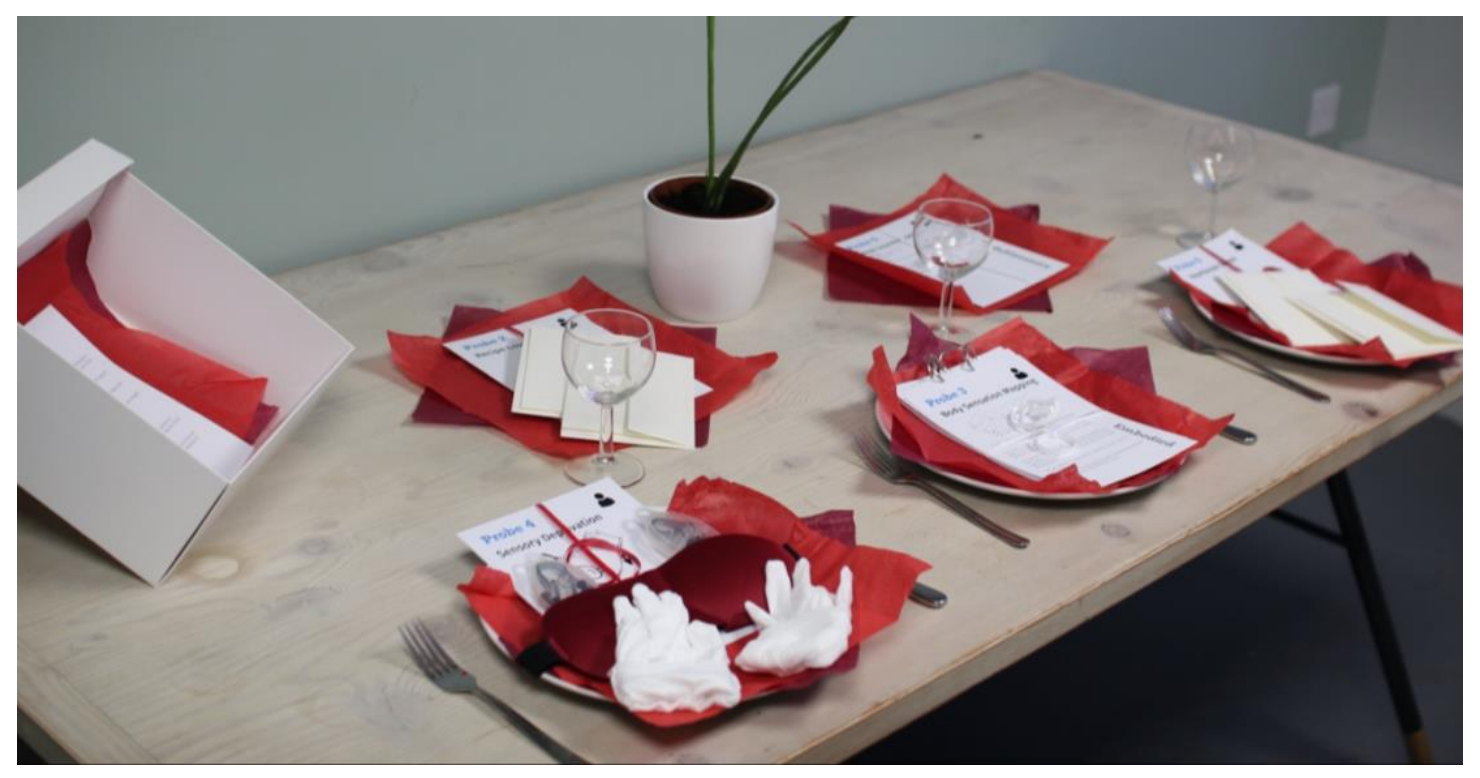

Figure 3 Package of sensory probes

\section{Sensory Probes}

The package (Figure 3) was conceived to be delivered in person to participants in a box, for use in the food-emotion study. The main box was a plain white cardboard box 245 x 245 x $116 \mathrm{~mm}$ with participant's name handwritten on top. The intention was to associate the package of probes with the design aesthetics of personal gifts in order to drive engagement by signaling to participants this was special, although participants were explicitly briefed that materials were to be collected by researchers at the end of the study. Gifting experiences with food has been suggested in the workshops, for example with references made to boxes of chocolates. Once the box was opened, the probes could be seen layered with instructions between tissue paper, further echoing the unboxing experience of food gifts. The probe instructions and gameboard design are available as supplementary material.

\subsection{TasteWho Gameboard}

The aim of this probe is to familiarize and sensitize participants to the depicted flavor terms, and prompt them to explore the matching of foods with their feelings or memories. The probe consists of a gameboard (400mm x 400mm), color printing images on card backing with overwrap finish, and folded in quarters. As shown in Figure 4 , the gameboard has 16 squares, each showing an abstract illustration for each flavor term, for instance "hot" is shown as a triangle in warm red/orange hues. Bespoke illustrations of the 16 flavor terms (including 5 for the basic tastes) were provided in order to communicate them not just through labels, but also visually. The terms were drawn from descriptors of flavor used in sensory evaluation [41, 63 ] and included basic tastes as well as bipolar descriptors such as " "warm" and "cool" for temperature. In order to support engagement and discovery of novel flavor terms by participants, the illustrations were purposefully abstract to generate curiosity and interpretation. Watercolor was used to generate these illustrations, inspired by prior work on illustrating smell, a related chemical sensation, by Kate McLean [40]. The illustrations were produced by the first author and revised through conversations among the research team through several iterations. In the Foodemotion study, participants were asked to select several foods from their kitchen cupboards that matched their emotions. Then, in turn, each couple's partner, conspicuously ate one of the selected foods, i.e., Piri-Piri sauce, followed by placing counters on the corresponding flavor labels on the board, i.e., "spicy" that matched that specific food, while the other partner attempted to guess that food, based upon the chosen labels. In the Food-memory study, participants played alone 
using this as a reflective activity to practice tasting and description of food. Participants were instructed to select foods related to their memories. The performance of eating and evaluating food supported a playful engagement of each participant with the experience of flavor.

\subsection{TasteWho Gameboard Design Iterations}

For the taste and flavor perspective the intention was to create a playful probe based upon the board game 'Guess Who' [66]. This approach borrows from a check-all-that-apply technique used in sensory profiling, in which various terms are presented to participants to indicate if the term applies to the flavor they eat [이. In this way, players could be introduced to a broader range of terms describing flavors. The initial design of the gameboard was created using words and abstract illustrations to support the understanding and engagement through both images and text, by drawing on synesthesia-informed approaches [42].

To evaluate the matching of each abstract illustration with each label or descriptive term, 25 initial word-image pairs were freely matched by 11 participants (v1, v2, v3, v4 boards can be seen in Figure 4)., Word-image pairs with over 50\% correct matches were kept for the second iteration of the board, i.e. , spicy, crunchy, sharp, , cool, while the rest were revisited to purposefully include basic tastes: 'bitter', 'sweet', 'salty' and 'sour' alongside 'creamy' to support texture description; while 'bland' and 'burnt' were selected to describe absence of taste or unpleasant flavor.

The $2^{\text {nd }}$ version of the TasteWho gameboard was evaluated in the same way as the $1^{\text {st }}$ one, by 5 new participants who had not taken part in the initial evaluation. Word-image pairs with over 50\% correct matches were kept for the third iteration of the board, i.e., floral, sour, burnt, salty, sweet, while bitter, hot, creamy and soggy were given new illustrations. The shapes for "Bitter" taste were made spikier to better capture its metaphoric meaning. "Hot" taste was illustrated through a triangle in red/orange hues. This contrasted better with its opposite flavor "Cold" illustrated through a triangle in cold blue hues. "Creamy" flavor was given a more curved form to represent the physical mechanics of creamy foods while the color for "Soggy" illustration was desaturated. A final evaluation of the gameboard took place with 10 older adults (over 65, all female) who were part of an arts and crafts group for older adults. They were given the v4 board with 'Warm' used to oppose 'Cool' more directly and 'Savory' replacing 'Umami' as a more commonly understood, equivalent term which largely matches the experience of umami.

Grounded in these findings, the final design of the TasteWho gameboard (Figure 4, v4) included in the 16 illustrations those for the five basic tastes: sweet, salty, sour, bitter, savory (instead of umami); three for texture: crunchy, soggy, creamy; two for temperature: cool, warm, and six for specific flavors: spicy, savory, floral, burnt, sharp, bland. The abstract stylized images matching these terms varied in color and shapes: pastel warm colors such as pink or blue for "floral" and "sweet" also represented through round petal-, and candy-like soft rounded shapes, respectively, and red color for "spicy" also represented through sharper angular forms inspired by 4 red chilies positioned in a straight cross. While "warm" was represented through warm colors such as orange and red, "cool" was shown through cooler colors such as blue or grey. 

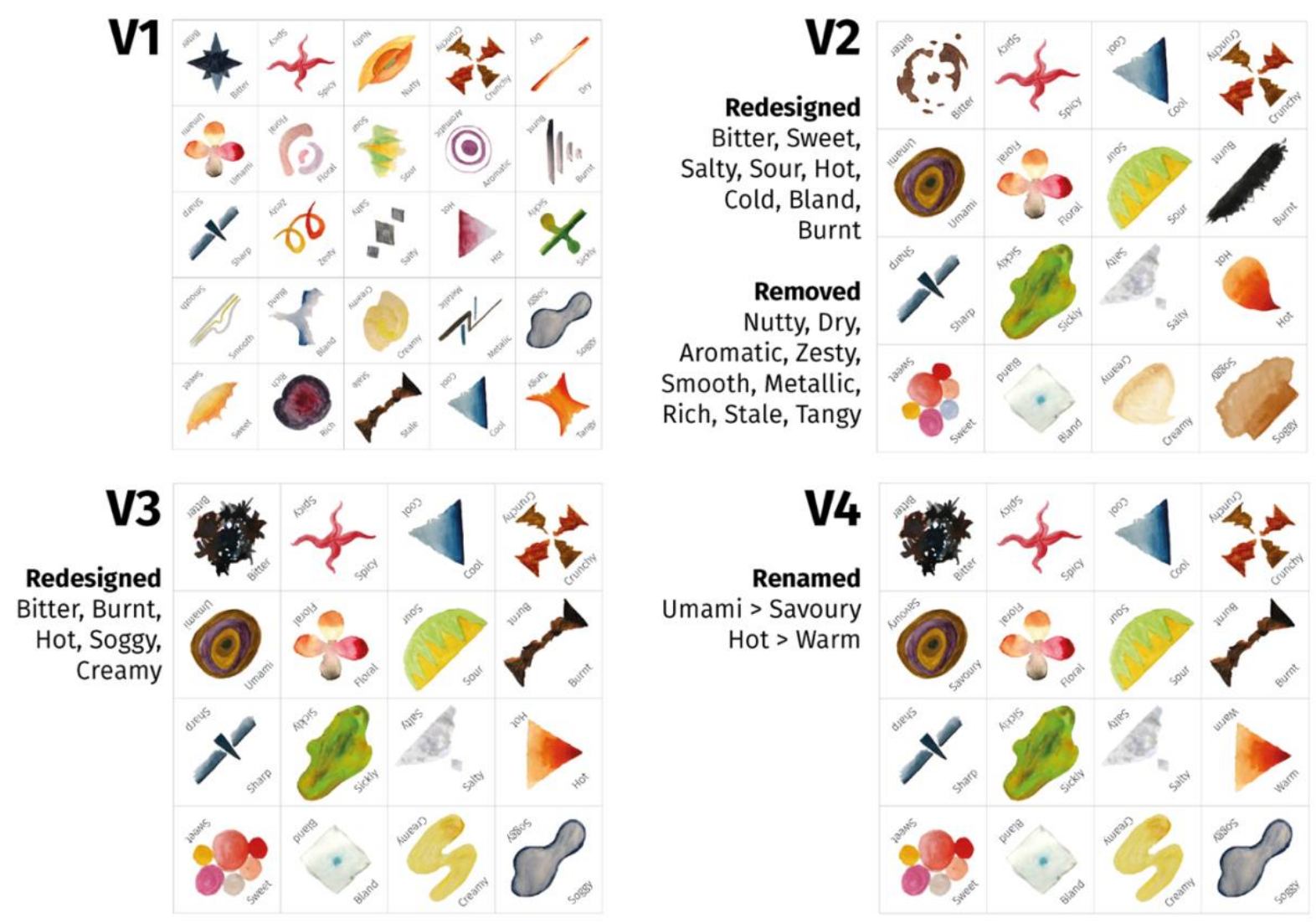

Figure 4 Taste Who Gameboard iterations V1-4, with annotations describing changes between versions 


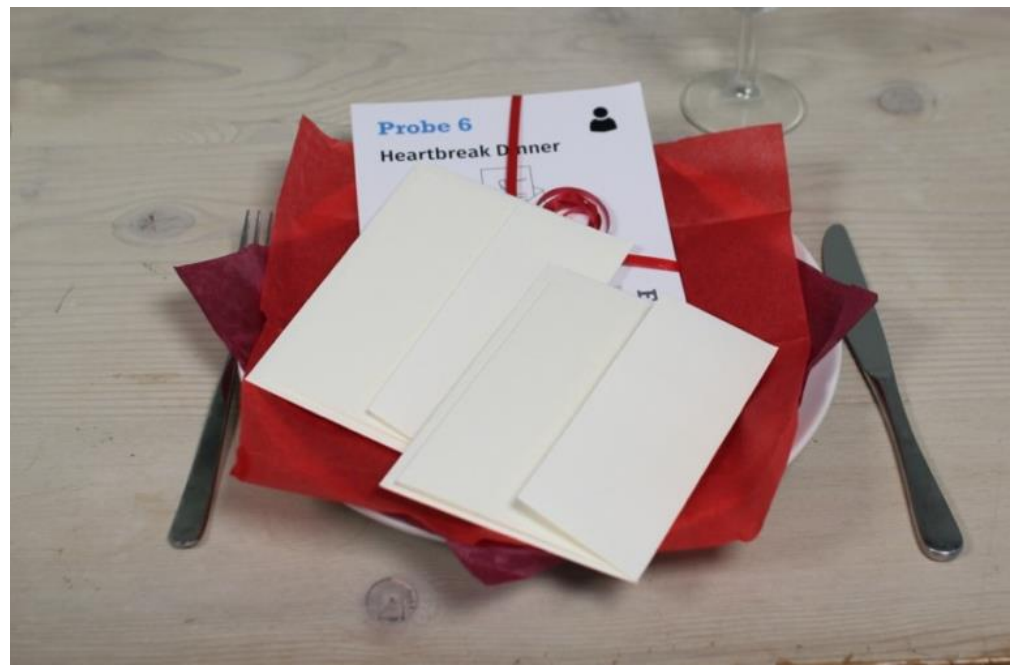

Figure 5 Writing recipes as Letters materials

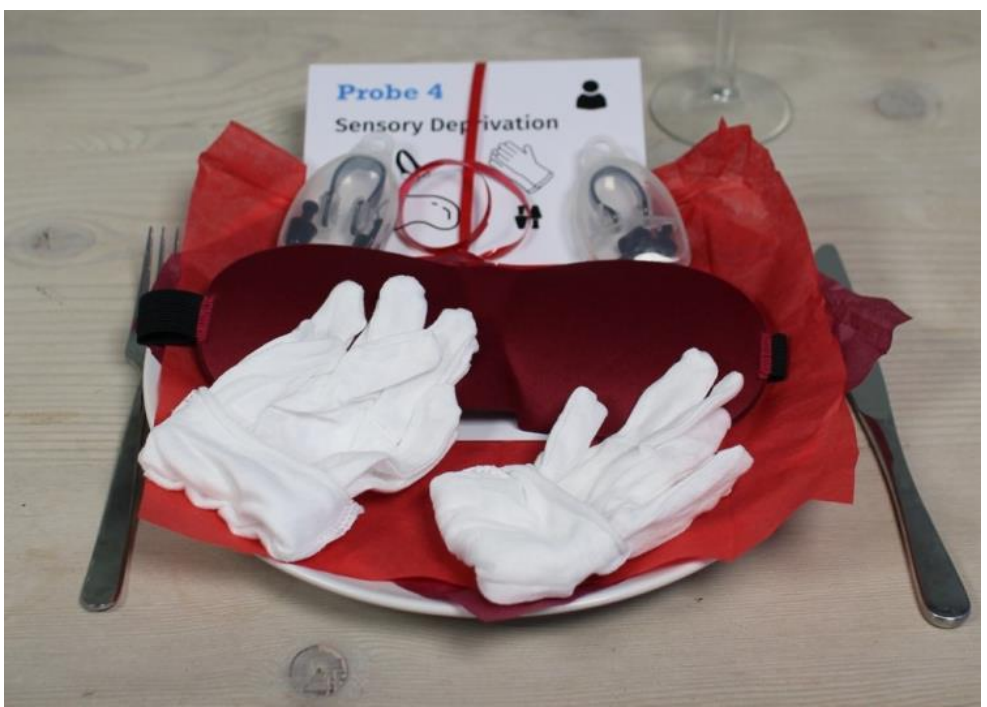

Figure 6 Sensory augmentation probe materials

\subsection{Writing Recipes as Letters}

The aims of these two probes were to support participants to label and associate foods with different meanings in relation to specific memories or emotions. Participants were asked to write, on given letter papers, recipes to represent emotions or memories (Figure 5). In the Food-memory study, one of the probes requested a love letter to one's partner including a recipe, while the other probe requested a letter to oneself describing a recipe to match the experience of being heartbroken which was later burned and whose food was not to be eaten. This act of catharsis was intended to disconnect food from its normal function of nourishment. In the Food-memory study, participants wrote a letter including a recipe representing a memory with food, and another letter with a recipe for a memory without food. 


\subsection{Sensory Augmentation Probes}

The aim of these 4 probes (Figure 6) was to increase awareness of the role of different sensory systems in the experience of flavor. Participants were instructed to eat an everyday, familiar meal while sequentially using each of the 4 sensory augmentation/deprivation probes in turn, namely blindfold, earplugs, nose clip, and gloves so that could not see, hear, smell or directly touch the food. After use, they described the impact of each probe on cards. This probe was instructed to be used in the same way for both Food-emotion and Food-memory studies.

\subsection{Body Mapping Booklet}

The aim of this probe was to increase internal or interoceptive perception related to the experience of digestion and metabolization. Participants were given a booklet and instructions to record feelings at three moments in time: immediately after, 30 minutes after, and 2 hours after eating an everyday meal. The choice to include the temporal unfolding of these experiences was inspired from workshops' findings on the feelings of fullness or relation between spicy taste and anal sensations. For each of these time intervals, participants were also provided with 4 cards depicting body outlines for different aspect of internal experience: mouth and tongue, stomach and gut, brain and nervous system, and heart and circulatory system (Figure 7). Participants were asked to write or draw on the cards their either explicit or vague experiences in relation to each bodily system. This probe was instructed to be used in the same way for both Foodemotion and Food-memory studies.
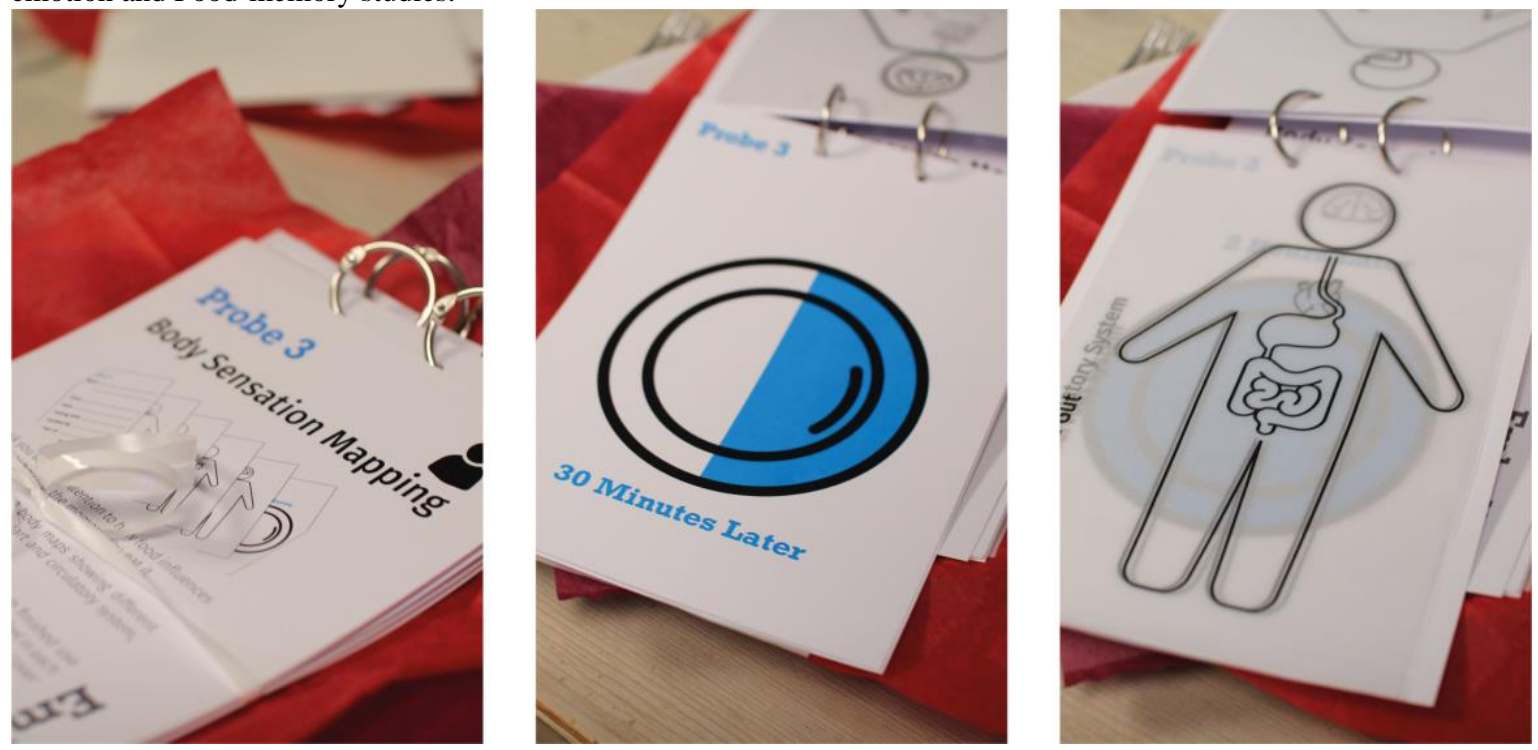

Figure 7 Body Mapping probe parts

\subsection{Probes for Capturing Environmental Context}

The aim of this probe was to gather insights into, and direct attention towards the environments where eating takes place. Participants were instructed to collect audio recordings and take photos both of the places where they made food and consumed food, showing the food in each of these contexts. In the Food-emotion study, participants took photos using smartphones, as a collaborative activity performed by the couples, while in the Food-memory study, they were provided with audio recording devices and disposable cameras. 


\subsection{Food-Emotion Diaries}

The aim of this probe was to support the capture of insights into relationships between foods and moods, and prompt reflection on them. For two weeks, participants photographed everything they ate, who made the food, who they ate it with, and their mood at the time, on a bespoke webform where date and time were automatically recorded (Figure 8). Visualizations of such data were generated to compare foods by mood, the maker of the food, and the social context of eating (Figure 9). In the Food-emotion study, these visualizations were individually reviewed by each participant through workshop with the first author to uncover insights into their food experiences. Participants were asked to review foods sorted by time eaten, whether eaten socially or not, and the associated mood. They were prompted to describe any identified patterns and to discuss any dishes in which they found surprising matches between food and mood, social context, or time of day. These interviews took about 15 minutes.
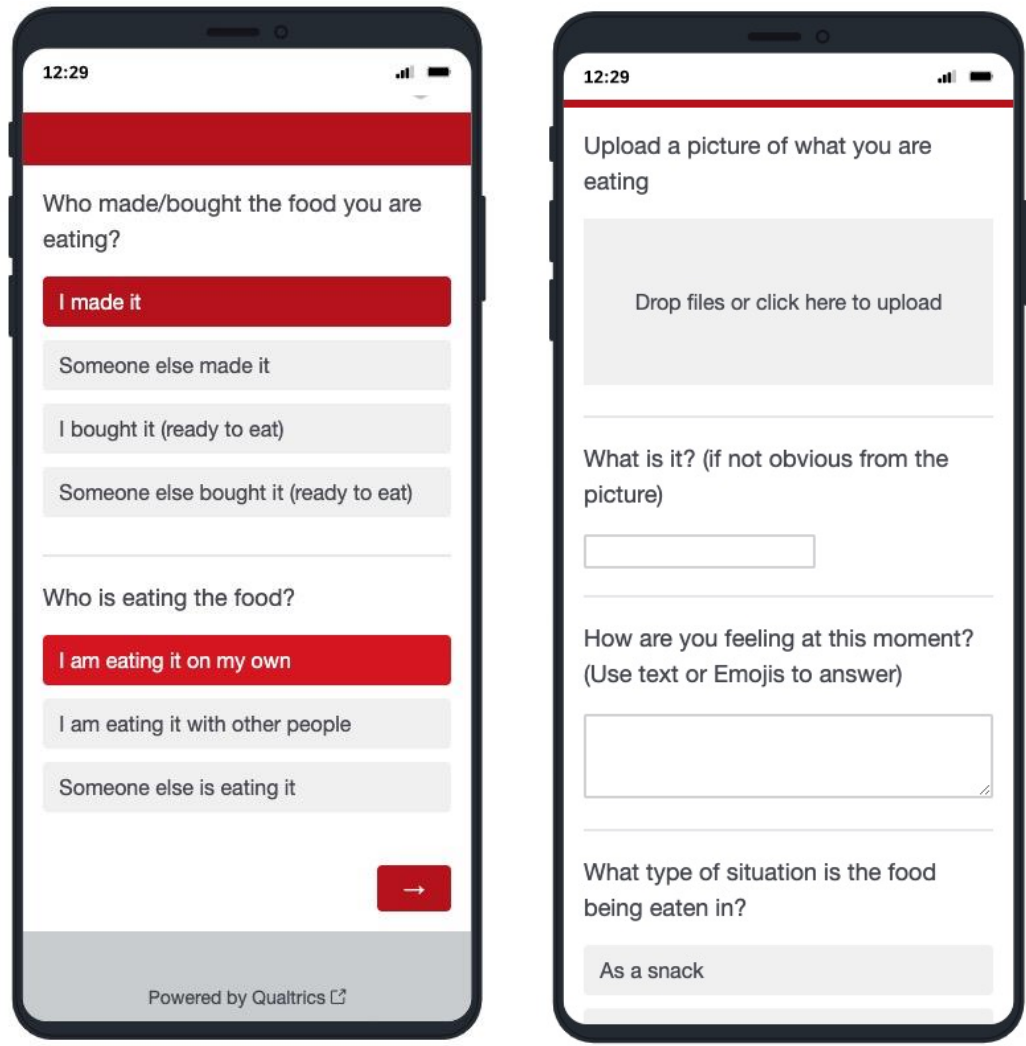

Figure 8 Food Diary recording screens 


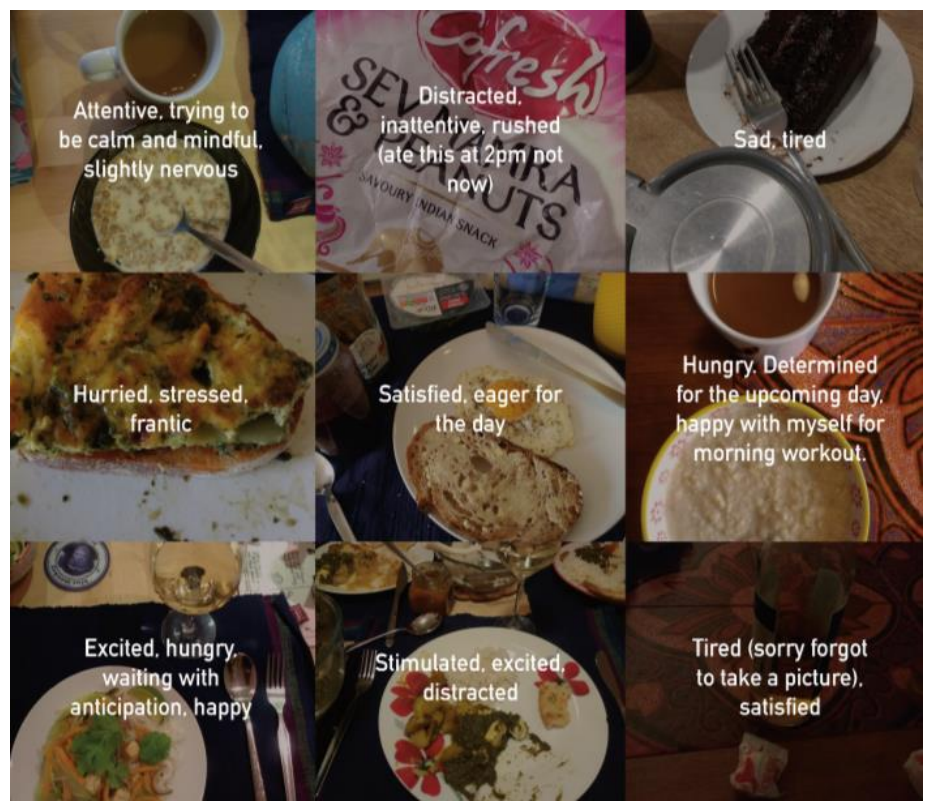

Figure 9 Detail from a participant's food diary visualisation Food-emotion diaries

\section{Diary Studies for Probes Evaluation: Method}

Following the design of the probes, we run the two user studies focused on food-emotions, and food-memories, respectively, with the aim to evaluate the probe package and further improve it. The sensory probes were used for two weeks by 10 participants (P1-P10) (5 couples: 6 female, 4 male, adults under 60) in the Food-emotion study. In the light of this study findings, the probes and in particular the TasteWho Gameboard were slightly revised and used for another two weeks by 8 participants aged over 60 (P7-P14) (6 female, 2 male) in the Food-memory study. Participants in both studies were recruited through convenience sampling via adverts on social media. The focus was not on experts such as chefs, but to recruit ordinary people, while ensuring that both younger and older adults are represented, given the impact of age on perception of flavor. The overall sample of 18 participants across the two studies is adequate for similar diary studies and provided a rich data set. In each study, participants were also interviewed after the collection of the probes. The interviews explored overall experience with the probes, whether it was enjoyable or challenging and why, and about their experiences of each specific probe. The interviews were audio recorded, fully transcribed and analyzed using a hybrid approach [17]. The thematic analysis involved both deductive codes such as identifying disruption,

destabilization, emergence and embodiment [65] and inductive ones emerging from the data such around the experience of using the probes, and in particular bodily and sensory experiences. .

\section{Findings from Probes Evaluation Studies}

\subsection{Overall Experience with Sensory Probe Package}

An important finding is participants' strong engagement and delight with the sensory probe package. Fun was the most commonly description of how all probes were experienced ( $\mathrm{n}=5$ participants): "it was enjoyable [...] because it was something that I'd never done before [...] it was sort of an anticipation of what's going to happen now" (P14, FoodMemoryStudy). Novelty supported a sense of playful discovery as highlighted by P17 (Food-Memory Study): "you do it with a spirit of [it being] a game, you're playing [...] so that was quite fun". Enjoyment was also derived from tasks that 
prompted activities found pleasurable such as $\mathrm{P} 1$ who "liked the process of writing a Letter [...] because that is being lost [...] I thinkit is nice also receiving a physical thing”. Engagement $(\mathrm{n}=4)$ with the probes was also supported by the box being "beautifully presented [...] I really enjoyed opening the box, I found it was so exciting the way you laid it out, you know with all the tissue paper and it was really like, a really an experience for both of us to like explore it all which was really nice" (P1, Food-emotion study). This supports the use of gift aesthetics in the packaging of the probes, that could shape the overall experience through an initial positive reaction.

Such enjoyment directly led into engagement with the sensory experiences facilitated by the probes: "[the probes were] fun and quite interesting. I found that some of the [sensory augmentation tools] really help you taste the food, and some detract" (P17, Food-Memory Study). The probes were not only seen themselves something "fun to receive" (P15) but through their use, they led to moments where participants "enjoyed thinking about food, we did [the TasteWho probe] together" (P17, Food-Memory Study). Interestingly, partner's engagement with TasteWho boardgame was not required for the Memory study which P17 took part in, yet both P17 and P15 spontaneously used this probe with their partners "[my] wife was preparing the five samples, and then [we were] sitting and actually tasting something and thinking about what you're tasting rather than just eating it [was enjoyable]” (P15, Food-Memory Study).

\subsubsection{Bodily experience of food.}

Both the Sensory augmentation and Body mapping probes aimed to provide new perspectives on multisensory experience regarding interoceptive experiences of food, which helped make salient experiential details usually unapparent $(n=5)$, as described by P14 (Food-Memory Study) who reflected on how the probe led them to think more deeply than they would usually: "it made me think really. Often, the only thing [I notice] is, it's a lovely, you can taste it, you think it's very nice or you're not happy with that flavor or whatever. But you don't actually think of how all those feelings going down or all those senses. So [the Body mapping] was, you know, it was a bit difficult, but it was useful". This challenge was welcomed by P2 (Food-emotion study) who described the Body mapping probe as "making yourself more aware, because you are talking about different parts of your body, that made me think about how you are supposed to chew things a certain amount of times". In this illustrative quote participant is prompted to consider not only the experiences as they occurred but also how the way he eats could impact on that experience. For some participants these new bodily insights were surprising: "weird; I had not done something like that before [it was] quite interesting [but] difficult" (P11, Food-memory study). This indicates the challenge of using these probes for taking on a new bodily lens to understand the food experiences taking place within the body.

Sensory deprivations were described by reliance on metaphor connecting the experience with "having a bad cold" (P12, Food-memory study) or "eating inside a container" (P13, Food-memory study). The different objects in this probe exposed different experiential aspects: "[with] the nose clip, I found that a bit difficult to swallow, as well as not smell it, so I didn't get the same sort of feeling about the food [as normal]. The blindfold it was it brought home to me that it must be really hard for [visually impaired] people to start to imagine what's on your plate. [...] I found that it was really hard to forme to really enjoy the meal because it was more trying to work out how to eat" (P14, Food-Memory Study). These different facets of food experiences accessed through sensory augmentation probes were a common theme, reported by 13 participants. Interestingly the above illustrative quote connects together immediate sensory changes with more behavioral observations around how food might be eaten by different people or in different ways. New ways to understand food experiences also emerged from other probes, including the Letters which were based on "the link between food and memory, which you kind of know it [in terms of] theoretically knowing. But, being asked to commit quite a bit of time to it, makes me understand the link much more clearly" (P17, Food-Memory Study). The Letters in the Food-emotion study also required participants to cook the food whose recipes have been written, thus extending the engagement with the food experience beyond eating and into the cooking practices.

\subsubsection{Sense-making through narrative construction.}

In both the Letters probe and the food-emotion diary reflection, participants constructed narratives through food. In the Letters, participants started with an experience and connected them to a foodstuff. These included some letters requiring imagined narratives and some that requested simple reconstructions of food eaten at the time. In contrast, for the diary reflection, participants started with the food and worked to recreate the narrative of the specific experience or to construct a broaden narrative connecting several foodstuffs through a theme as seen in this quote by P7 (Food-emotion 
study) "I would put these [foods] in the same camp [...]I maybe didn't like them as much, they are a bit stodgy, that is why. [Whereas] you definitely see the positive emotion with the healthy stuff". The probes worked to seed the idea of food as a meaning making tool: "the whole thing has been quite an exercise in excavating memories and thinking about them, and also thinking about the part that the food plays in daily life, obviously [and] the link between food and memory" (P9, Food and memory study).

To conclude, the overall package was perceived as a whole, in which each of the probes captured different aspect of food experience, from flavor and multisensory aspects to external contexts. The design of the probes to include a range of tasks involving different bodily, emotional and cognitive activities while connecting with participants' eating experiences has been key to ensure this cohesion of the probe package. They also complement each other as they support distinct aims from those capturing food experiences, to those exploring embodied experiences and those for facilitating reflection towards shared understandings of such experiences

\subsection{Experience of Individual Probes}

\subsubsection{TasteWho Gameboard.}

The probe ( $\underline{\text { Figure 10 }})$ was seen as easy to complete ( $\mathrm{n}=4$ participants) and fun $(\mathrm{n}=4)$ as shown int the following quotes: "the one where I had to put a counter [TasteWho gameboard probe], that was easy. Very beautifully designed" (P18, Food-Memory study), "it felt like it was a game, and it was fun. It was light to do and yeah, it was enjoyable" (P17, Food-memory study). P7 identified this probe as their "favorite [...] I'd like more interactive ones like that, fun and interactive". This probe's primary impact was to prompt attention to the experience of eating (n=6), disrupting the act enough that participants could become aware of its details: "everyday taste [...] you take it for granted, you are generally just eating without thinking, it makes you think. For an example this chocolate, I can just go and buy some chocolate [...] I would not even give it a thought, is it burnt? is it sweet? is it bitter? Yeah, so I think this exercise is very fruitful" (P10). This is an illustrative quote indicating increased awareness to the different sensory experience of flavor and taste, as reflected by most participants $(n=7)$. The choice of foods used in this probe was also seen as important $(n=3)$ with a few participants suggesting the use of less similar food descriptors such as bittersweet and meaty ( $n=6)$ in order to avoid the experience becoming repetitive $(n=4)$.

\subsubsection{Writing Recipes as Letters.}

This probe disrupted the mundane experience of preparing food by drawing on strong emotional experiences and how to express them in different modalities through written recipes and therefore flavors and tastes: "it really makes you think about eating and specifically think about certain meals that would be like romantic for your partner" (P2, Food-emotion study). The heartbreak Letter and recipe were particularly interesting as they challenged the common association between food and positive emotions: "the heartbreak one I found really disgusting because I left this chicken for like ages and it just, it was probably a bit dangerous being in the fridge and being so over passed, it made me feel really repulsed. Which is maybe how I feel about [the relationship]" (P2, Food-emotion study). Participants in the Food-memory study noted that "the first recipe was really easy, the other one wasn't quite so easy, where you had to [make a recipe for a memory without food]" (P18). 5 participants described the Letters as difficult: "I've got a bit sort of bogged down in [doing the Letters. But then] a scorching image came up for them" (P16, Food-memory study). The process of completing the probes, particularly in the case of the Food-emotion study resulted in other benefits, i.e., meaningful gifting like in the quote "I found the process of making you [one's partner] something I had never made you before, it kind of expressed my feelings, it wasn't so much, I didn't make it in a heart shape, it was more than I know you are going to really enjoy this and it has taken a long time to do" (P2, Food-emotion study). 


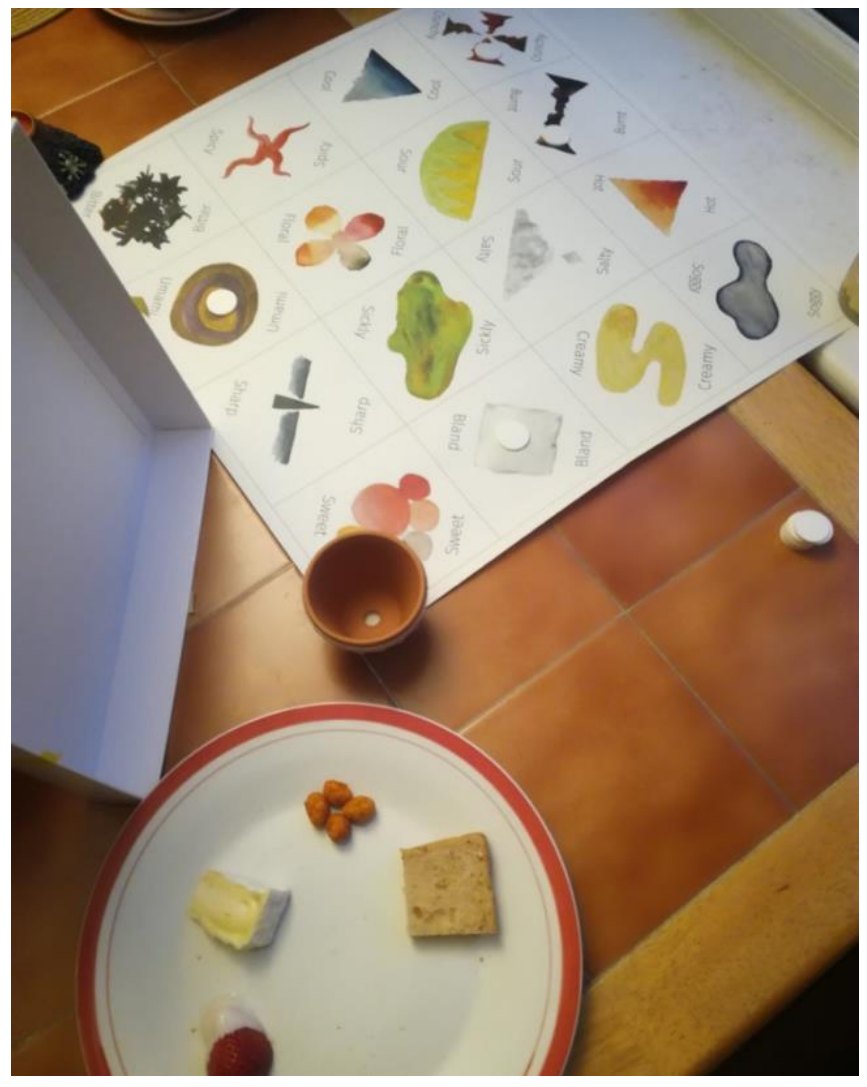

Figure 10 The TasteWho Board in use with one participant's food on a plate and counters on the board to describe the taste

\subsubsection{Sensory Augmentation/Deprivation Probes.}

This probe consisted of four artefacts that changed bodily sensations [65], disrupting the normal experience $(\mathrm{n}=5)$ and fragmenting the compound flavor experience, so that other sensory elements could be better appreciated: "[I discovered that] the whole connection between smelling and tasting is important, eating something without being able to smell it, is obviously quite a different experience, [...] eating blind, that was quite an odd one [...] more of manual dexterity challenge. You know, 'where's the fork going?', 'How's the sound of the plate?', that was my overriding sensation. It was more about how I actually, mechanically got the food into my mouth. The sensation that gave me the greatest experience of taste was wearing earplugs and not being able to hear anything. That was the one that really heightens my sense of taste" (P17, Food- memory study). 4 participants identified this probe as giving them a new perspective on food experience: "the smell of food, like cooked food or hot food. That's so much part of the experience is the smell. I mean, when I did the sensory probe, it's quite strange [I realized that] when you do block off the smell, you really could be eating anything" (P11, Food-memory study).

With respect to visual sense, $\mathrm{P} 7$ found similarly that "you appreciate the presentation a little bit more as well, when you're blindfolded". However, these insights were not shared across participants, reflecting a rather idiosyncratic experience from interacting with these probes: "I found this test particularly unrewarding with each deprivation. I could not identify any notable observation or changes to the experience" (P15, Food-memory study). One explanation for this may be the quite detailed understanding this participant already had of how smell worked, which biased against appreciation of the differences: "I was conscious of when stopping my nose from smelling, I'm aware that most of our 
taste is done through the nose. But I'm also, I was also conscious that while the food was in my mouth, it was still getting to my sinuses through the back of my nose. So, I was still being aware that there was a smell" (P15). This indicates that probes are most useful for participants who have less technical understandings of, for example, retronasal olfaction. Participants also found the nose clip uncomfortable, this discomfort disrupting the ability to focus on the changes to flavor experience (n=3) "the nose clip, I couldn't put it on, it was tiny. It was very painful” (P15, Food-memory study).

From this process there were many reports of emerging connections between food and other forms of experience $(\mathrm{n}=7)$, "It made me think [...] that I'd like to go on a meditation retreat, eating in silence." (P16, Food-Memory Study). In terms of hearing sense, 2 participants felt it had the strongest impacted on their experience of food: "the earplug one that was the strongest experience, I found the most fascinating [...] just focusing on the experience of eating” (P16, Food- memory study). A few participants identified some of these artifacts as challenging. For instance, 3 participants felt that the nose clip has changed how they ate, as it made it "difficult to swallow" (P6, Food and memory study) while the blindfold led to "difficulty in eating with your eyes" (P7, Food and memory study). Overall, these probes succeeded in exposing participants to novel sensations ( $\mathrm{n}=4)$ :" it did feel a bit sexy, in a weird way" (P2, Food and emotion study) while providing novel perspectives on the multisensory experience $(n=4)$.

\subsubsection{Body Mapping Booklet.}

Body mapping disrupted the familiarity of eating: "you are being so aware of your [...] normally I just eat it and think nothing of it, I think about the taste, but I don't really think about how it is making my body feel later" (P1, Foodemotion study). In some cases, participants struggled to notice any impact on their body, a key reason being that the probe's activity was perceived as difficult $(n=6)$. In turn, 2 participants consider particular foods that would result in stronger internal responses: "I should have over-eaten rich foods that have made me feel sick or something" (P8, Food and memory study). P15 also felt that he "couldn't really find very much to it [even though he] loved the little drawings [I was] so frustrated that there wasn't any data to record". Most participants chose to write any observations they had, with only two opting to draw. All participants had fewer observations over time, with very few recording any noticeable experience after 2 hours. 2 hours had been selected to provide time for the gastric emptying [39], covering the time food spent in the stomach. However, experience at this stage may not be apparent unless it is unusual, in either compositionor amount. Despite some challenges this probe was still seen as providing novel perspectives (n=3), "[It made me] more aware, because you're talking about different parts of your body" (P2, Food-emotion study).

\subsubsection{Recording Probes for Capturing Contexts.}

This probe was found to disrupt eating experience to a large extent, impacting on the ability to capture details of experience as they would normally be, "I think knowing that I was being recorded [meant] I wasn't saying anything [... I thought] I sounded boring as hell, [...] I felt intimidated” (P9, Food-emotion study). The aim of this probe was to support the capturing of the environmental sounds, which led to a shorter recording period chosen for the Food-memory study. But there were issues with the ability to use bespoke recording devices that required buttons to be pressed: "it is tricky trying to record what you're eating, or cooking is tricky when you've got to hold it down" leading to P2 (Food and memorystudy) to suggest recording "on the phone and [then sending] the sound clip". Overall, this probe was less successful in providing new perspectives on food experiences, aside from the intrusion that recording a dining situation had. While these probes closely matched the recording devices in Gaver's original cultural probes [18], future work is needed to understand the value of sound recording for sensory probes.

\subsubsection{Food and Emotion Diary.}

Participants described the act of recording as disrupting the habits related to food ( $\mathrm{n}=2$ ), prompting them to better consider their choices: "[I am not eating it] because I want it. Yes. But just because things are not going very well. And so that made me think well, this is not the answer" (P3, Food-emotion study). When reflecting on the data collected in the diary, what emerged were insights into decision making process, emotional experiences and connections between various social and bodily experiences and food: "since doing this [the diary] it has made me realize that my food consumption I was just getting takeaway" (P1, Food-emotion study), "we had stir fry's and things like that which I would never normallyhave but it is because whenever we meet up we tend to make different dinners" (P2, Food-emotion 
study). Whilst there is extensive work in HCI on tools for supporting healthier eating through tracking, this probe instead made habits more apparent.

Through the process there were many examples of participants uncovering habits such as foods eaten in social contexts vs when alone $(\mathrm{n}=7)$ that they were unaware of, which led to them reflecting upon behavior and choices such as foods eaten when tired $(n=5)$. Both the uncovering and reflecting were important actions to be stimulated by the probes to support the following design process. This probe also supported enacting a storytelling through food: "in fact, this week, if I remember rightly it was leading up to quite a big presentation I have to do, so there may have been more negativethings than not" (P2, Food-emotion study). This probe also provided opportunities to learn how food can act as a memory cue $(\mathrm{n}=4)$, "[I had] a friend visiting [...] I can see a scone that reminded me of that" (P5, Food and emotion study), and revealed the social aspects of shaping food experience "I mainly do the cooking and I think it is really hard because, whenever we are in his house we are in a shared kitchen but when we are at my place, I don't have an oven, so we are so limited in what we cook at my place" (P1, Food-emotion study).

\section{Discussion}

In the light of our findings, we now revisit the initial research questions, starting with reflection on the sensory probes as a novel design research method that extends in new ways the affordances of traditional cultural probes. In particular, they prompt the capture of sensory fragments of experience through both internal and external senses, by extending the engagement of the body in this capture beyond visual and aural modalities to include also taste, smell and interoceptive senses involved in the ingestion and digestion of food. These bodily-based affordances generate ambiguity which fosters novel perspectives on food experiences and contribute to the playfulness characterizing the engagement with sensory probes. Our probes are exploratory design research methods which similarly to Gaver's cultural probes $[\underline{18}, \underline{19}]$ are intended to provoke participants and engage them in a dialogue with probes materials whose data can then inspire designers. Our probes' novelty resides in their specific focus on participants' sensory and bodily experiences. We explored how participants understand and engaged with them, and how probes were revised to better support such understanding, while further work could look into how such the data collected through the probes can inspire both designers and users to co-create personalized flavors. For instance, we have already used probes' data to co-design personalized flavors with the 8 couples after they took part in the diary study. Our couples used these personalized flavors in their homes to communicate emotional content and support co-regulation of emotions in their intimate relationships [23].

\subsection{Affordance of Sensory Probes for Capturing and Increasing Awareness of Food Experiences}

We co-designed the sensory probes, a novel design research method inspired by the cultural probes [18] with the aim to understand the richness of food experiences by sensitizing participants to their sensorial, emotional, cognitive and social aspects. Among these aspects, it is the purposeful emphasis on sensorial and bodily aspects that sets sensory probes apart from cultural ones, as further detailed.

\subsubsection{Capturing sensory fragments.}

An important affordance of probes is that of capturing participants' experiences or what Graham and colleagues called fragments [25]. These are snippets or slices of personal experiences usually in the form of photos, illustrations, brief audio recordings or written notes on cards. In contrast to cultural probes, sensory probes sensitized participants to capture what we call sensory fragments where the sensory and bodily aspects of the captured experiences come to the fore, while accounting also for their emotional, cognitive, and social aspects.

Among sensory probes, those that particularly supported the capture of sensory fragments were the four Sensory augmentation/deprivation probes and TasteWho gameboard. In addition, the Body mapping booklet also emphasized the role of body in food experiences both during, and after food was consumed. The probes supporting emotional, cognitive, and social aspects were Letters, Diaries which also allowed the construction of meaning, as well as TasteWho gameboard 
with its playfulness quality. Finally, the sound and camera recorder supported the capture of external socialand spatiotemporal context, similar to other similar artifacts part of cultural probes [18].

Probes for brief sensory augmentation/deprivation facilitated experiences in which sensorial aspects such as visual, aural, olfaction or direct touch were turned on and off. This has led to sensorial deprivation of that specific sense and in turn, to sensory augmentation of the other senses [32]. TasteWho gameboard involved eating activities which thus emphasizing all sensory modalities involved in food experiences. More importantly, our findings indicate the value of sensory fragments for bringing into awareness sensory and bodily aspects of food experience that otherwise would go unnoticed.

\subsubsection{Engaging interoceptive senses.}

Cultural probes have focused predominantly on first person experiences captured through external senses such as vision, hearing and touch $[\underline{18}, \underline{25}]$. Sensory probes innovatively extend this focus to include also experiences captured through internal senses such as those involved in the ingestion and digestion of food. These refer to visceral experiences such as that of chewing, fullness or how digestion unfolds over time [ 8$]$ which have been limitedly explored in interaction design. These allowed participants to gain access to important novel perspectives of their food experiences by slowing down, focusing, observing, and reflecting on them. The Body mapping probe has been particularly useful in supporting access to these interoceptive experiences, although some participants found it difficult to engage with. This is not entirely surprising, as such probe draws from interoceptive awareness which requires time to develop [13, $\underline{17}]$. Nevertheless, the body mapping probe could benefit from additional instructions to better scaffold participants' engagement with it.

\subsubsection{Engaging additional external senses: Towards flavor design worlds.}

As mentioned earlier, one of the key roles of cultural probes is capturing participants' experiences mostly through the external senses of sight, hearing and touch $[\underline{18}, \underline{25}]$. Interestingly, although a camera was extensively used to capture both food and context of food for the diary probe, the sound recorder was less used mostly likely due to therisk of capturing intimate commensality conversations. Given however the role of sound in food experiences [34,43], future research can explore how sound-based probes can be better designed to address these concerns. The other external senses of taste and smell have been limitedly leveraged in cultural probes. Our findings however have shown that taste and smell can be also successfully engaged through tailored probes particularly the TasteWho gameboard that prompted activities entailing food experiences, be them the act of eating food, preparing food, writing recipes to communicate emotions or memories, or of reflecting on these food experiences, either individually like in Food-memory study or in couples like in Foodemotion study. This gameboard also offered the opportunity to enrich participants' vocabulary of flavors and taste through visual and semantic labels of diverse food. Whilst our sensory probes do not contain food material per sei, they are each designed to engage with the food material around participants.

Furthermore, our design research method can be also contextualized within the emerging work in multisensory human-food interaction where for instance 3D printed personalized flavors have been used as material food probes as part of the design process of novel food interactions $[\underline{18}, \underline{23}]$. We argue that our sensory probes could offer a strong initial design research step for the development of such design tools involving food that can be used later in the design process of novel technologically mediated food experiences. Not at least, by capturing accounts of personal experiences integrating short narratives with rich sensorial details [48] from both phenomenological and social interactions with food [48] our sensory probes humanize [25]. Through an emphasize on flavor rich experiences, our sensory probes also contribute to extending Bartoshuk's taste world [] ] to what we call flavor design worlds highlighting the wider multisensory experience of food that goes beyond taste to integrate smell, vision, sound and touch, and which in turn can be used to inform the co-design of human-food experiences. Flavor design worlds are unique for each individual, consisting of sensory of fragments of rich food experiences that can be made available for design.

\subsection{Novel Perspectives on Food Experiences}

The mundane and ubiquitous aspects of food experiences can challenge people to notice and articulate them. For this, we leveraged the affordances of cultural probes employing ambiguity and mystery in order to shed light onto the taken for granted experiences [19]. We used the disrupt and destabilize stages of the embodied ideation framework [드] to inject 
estrangement and unfamiliarity in our sensory probes and their activities. Thus, we used three strategies to ensure new perspectives which are further detailed, strategies that have been limitedly used in cultural probes.

\subsubsection{Changing bodily sensations through sensory manipulation.}

Our sensory augmentation/deprivation probes including blindfold, earplugs, nose clip, and gloves were key for delivering sensory manipulation through brief sensory deprivation of each of the four main external senses which in turn allowed the augmentation of the others and particularly taste. These probes were inspired by object-led explorations such as those involved in the Sensual Evaluation Instrument [이 ] and active engagement with sensory manipulation as in A-labs [32]. Which of these four objects were most effective varied from person to person, some found that the earplugs had the strongest impact, in particular heightening the internal aural experience of eating. For some the nose clip was uncomfortable and to be discarded, whilst others found it useful. This highlights the importance of individual differences due to the perceptual thresholds that underpin sensory augmentations. This could be addressed by a range of such probes, so that people can explore them to select the ones that best match or complement their dominant perceptual style [52]. It is worth noting that even smaller sensory augmentation could be beneficial to explore. Unlike Gaver's probes [18] which aimed to search for fragments that could be inspirational, our sensory probes search for both presence and absence of experience. This opens up the design space for multisensory design to not only attend to whatis noticed or present, but also to what is missed or imperceptible to an individual. Sensory probes support an approach to design which recognizes the role of the individual in each sensory experience and thus moves beyond the more general approaches to which merely deconstruct and reassemble sensory experiences[14,42,56]. In contrast, our Sensory augmentation/deprivation probes place the multisensory experience within the context of the body perceiving it. They are not only about capturing personal data, but also about building individual's sensitivity towards their bodily experiences. What emerges from the disrupting and destabilizing [ㄷ] ] of the probes is not isolated insights but more likely participants' knowledge of their flavor design world that they learn to embody [65].

\subsubsection{Bringing awareness through bodily enacting of novel actions.}

The use of the Body mapping probe in both evaluation studies, and of the Letters in the Food-emotion study involved bodily enactment through which participants become more aware of their food experiences. Both probes ensured this by providing different ways to make the experiences strange. Participants were prompted to enact actions either by observing and articulating their internal sensations or by creating recipes for their partners, which some participants described as difficult. This difficulty relates to intentionally designed uncertainty [25] as part of these probes in order to prompt participants to complete difficult tasks that required cognitive effort and challenged assumptions. Uncertainty was also an affordance of the original cultural probes [18], however our sensory probes were explicitly crafted to disrupt bodily experiences in order to support the emergence of new understandings [ㄷ]. Our probes provoked [29] participants by enforcing certain ways of thinking and consideration of emotionally charged topics such as heartbreak or by physically augmenting their sensory capacities to experience food in a new way, similar to A-labs [32] and sensory bodystorming [62] methods. Although both Body Mapping and Letters involved bodily enactments with food, they were not only impacting on sensory experiences, but also on sense-making. While previous models of multisensory experience focus mostly on interaction[56], and the sensory experience required by it, sensory probes suggest a stronger integration of sensory experiences and sense-making,

\subsubsection{Recontextualizing and using food material in different ways.}

The TasteWho gameboard provided a space in which participants could playfully explore and engage with a richer vocabulary describing food experiences. Through the combination of text and image, the gameboard provided context for engagement where images were designed to scaffold the comprehension of novel flavor terms, but also as an invitation to play intended to inspire and aesthetically delight. This playfulness was diverse, experienced as cooperative-competitive by the couples in the Food-emotion study and exploratory by individuals in the Food-memorystudy. The play affordance of the cultural probes has not been much emphasized [18]. In contrast, our gameboard probe was designed so that food descriptions were neither fixed as might be the case with tasting notes on a bottle of wine, but more fluid evolving with the movement of the counters on the board. In this way the gameboard drew inspiration from the Sensory evaluation 
tools for non-experts such as check-all-that-apply [4]. The gameboard probe suggests thatmultisensory design tools which were created mostly for designers $[11,14]$, could also benefit users to better understand their bodily experiences.

\subsection{Lessons Learned by Co-creating the Probe Package}

The design of the sensory probes was grounded in the state-of-the-art of body-centric design, sensory evaluation, and multisensory methods, as well as in the insights gathered through the probe codesign workshops. Whilst previous work provided some framing $[\underline{28}, \underline{65}]$ and approaches to capturing experience $[\underline{60}, \underline{63}]$, it was through the personal experiential vignettes emerging from the workshops that provided most inspiration to create the probes. On reflection, the diverse materials and their modalities used in the co-workshops were key to open up the exploration of multisensory experiences through novel, bodily-centric probes.

The evaluation of sensory probes though two studies: food-emotion, and food-memory one, was beneficial for revising some of the probes. Thus, while Sensory augmentation/deprivation probes and Body mapping booklet remained unchanged, indicating their value across both evaluation studies, others were improved such as the Recording probes. The Letters and TasteWho gameboard were only slightly revised to match the topic of the second study. That these two probes could be easilyadapted to differing studies and contexts celebrating emotions and or the body, indicates they could be further adaptedto fit other emerging concerns in HFI such as space-based [47] or playful [2] food experiences. Despite their success in sensitizing participants and contributing to later co-design, there remains the potential to further improve these probes.

First, one common recommendation was more guided selection for the food to be eaten as part of a particular probe. In the design of probes (particularly Body mapping) the guidance was to engage with familiar foods reflecting participants' preferences in their everyday life. However, this resulted in a lack of new experiences over time, leading to participants' suggestions for large celebration meals that could provide more food experiences to engage with. Whilst this may result in a richer collection it may not provide added value. We argue that future work on sensory probes could focus on supporting better interoception, by better helping individuals to attune their bodily experiences. Such approaches can be explored through facilitated workshops on micro-phenomenology [ㄱ] or as part of somaesthetic interaction design approaches [28].

Second, some probes were too disruptive to the extent that rather than merely rendering an experience as unfamiliar or strange, the probes themselves become the main focus of attention. These relate to the sensory disruption such as the painful experience of the nose clip reported by a few participants, or the social disruption such as the intrusiveness of the sound recording during intimate meals. In these cases, the focus fell on the experience of use of the probe artefacts rather than experience of the food or flavor. Future work is needed to mitigate such unproductive disruptions.

\section{Conclusion}

This paper reports on sensory probes, a novel design research method which has been designed and evaluated through a codesign workshop and two user studies. This probe package successfully sensitized participants towards their food experiences both in relation to emotions in intimate relationships and memories in old age. Findings indicate strong engagement with the sensory probes and how they brought forward the bodily and sensory aspects of these experiences, alongside emotional and social ones. We highlight the new affordance of sensory probes for capturing sensory fragments, engaging interoceptive senses and external senses more broadly, and for enabling novel perspectives on food experiences.

\section{ACKNOWLEDGMENTS}

This work was supported by the Designing Multisensory Interactions: Emotional User Experience of 3D Printed-Food Consumption project (Grant Number 1962364) funded by EPSRC and Dovetailed Ltd. under a CASE PhD award and EC H2020 funded AffecTech: Personal Technologies for Affective Health, Innovative Training Network (722022). 


\section{REFERENCES}

1. Miquel Alfaras, Vasiliki Tsaknaki, Pedro Sanches, Charles Windlin, Muhammad Umair, Corina Sas, and Kristina Höök. 2020. From Biodata to Somadata. In Proceedings of the 2020 CHI Conference on Human Factors in Computing Systems (CHI '20), 1-14. https://doi.org/10.1145/3313831.3376684

2. Ferran Altarriba Bertran, Jared Duval, Elena Márquez Segura, Laia Turmo Vidal, Yoram Chisik, Marina Juanet Casulleras, Oscar Garcia Pañella, Katherine Isbister, and Danielle Wilde. 2020. Chasing Play Potentials in Food Culture: Learning from Traditions to Inspire Future Human-Food Interaction Design. In Proceedings of the 2020 ACM Designing Interactive Systems Conference (DIS '20), 979-991. https://doi.org/10.1145/3357236.3395575 3. Ferran Altarriba Bertran, Samvid Jhaveri, Rosa Lutz, Katherine Isbister, and Danielle Wilde. 2018. Visualising the Landscape of Human-Food Interaction Research. In Proceedings of the 2018 ACM Conference Companion Publication on Designing Interactive Systems (DIS '18 Companion), $243-$ 248. https://doi.org/10.1145/3197391.3205443

4. Gastón Ares, Cecilia Barreiro, Rosires Deliza, Ana Giménez, and Adriana Gámbaro. 2010. Application of a Check-All-That-Apply Question to the Development of Chocolate Milk Desserts. Journal of Sensory Studies 25: 67-86. https://doi.org/10.1111/j.1745-459X.2010.00290.x 5. Eshita Sri Arza, Harshitha Kurra, Rohit Ashok Khot, and Florian "Floyd" Mueller. 2018. Feed the food monsters! Helping co-diners chew their food better with augmented reality. In Proceedings of the 2018 annual symposium on computer-human interaction in play companion extended abstracts (CHI PLAY '18 extended abstracts), 391-397. https://doi.org/10.1145/3270316.3271520

6. L. M. Bartoshuk. 1978. The psychophysics of taste. The American Journal of Clinical Nutrition 31, 6: 1068-1077. https://doi.org/10.1093/ajcn/31.6.1068

7. Kirsten Boehner, Janet Vertesi, Phoebe Sengers, and Paul Dourish. 2007. How HCI Interprets the Probes. In Proceedings of the SIGCHI Conference on Human Factors in Computing Systems (CHI '07), 1077-1086. https://doi.org/10.1145/1240624.1240789

8. David A. Booth. 2013. Configuring of extero- and interoceptive senses in actions on food. Multisensory Research 26, 1-2: 123-142.

9. Rita Maldonado Branco, Joana Quental, and Óscar Ribeiro. 2016. Playing with personalisation and openness in a codesign project involving people with dementia. In Proceedings of the 14th Participatory Design Conference: Full papers - Volume 1 (PDC '16), 61-70. https://doi.org/10.1145/2940299.2940309

10. Wender L. P. Bredie, Hui Shan Grace Tan, and Karin Wendin. 2014. A Comparative Study on Facially Expressed Emotions in Response to Basic Tastes. Chemosensory Perception 7, 1: 1-9. https://doi.org/10.1007/s12078-014-9163-6

11. Serena Camere, Hendrik NJ Schifferstein, and Monica Bordegoni. 2015. The experience map. A tool to support experience-driven multisensory design. In DeSForM 2015 Aesthetics of Interaction, Dynamic, Multisensory, Wise; Proceedings of the 9th International Conference on Design and Semantics of Form and Movement, Milano (Italy) 13-17 Oct. 2015.

12. Claudia Daudén Roquet and Corina Sas. 2020. Body Matters: Exploration of the Human Body as a Resource for the Design of Technologies for Meditation. In Proceedings of the 2020 ACM Designing Interactive Systems Conference (DIS '20), 533-546. https://doi.org/10.1145/3357236.3395499 13. Claudia Dauden Roquet and Corina Sas. 2021. Interoceptive Interaction: An Embodied Metaphor Inspired Approach to Designing for Meditation. In In Proceedings of the 2021 CHI Conference on Human Factors in Computing Systems (Yokohama, Japan) (CHI '21).

14. Claudia De Giorgi, Beatrice Lerma, Cristina Allione, and Eleonora Buiatti. 2011. Sensory evolution: sensory and sustainable design strategies. In Proceedings of the 2011 Conference on Designing Pleasurable Products and Interfaces (DPPI '11), 1-4. https://doi.org/10.1145/2347504.2347512 15. Markéta Dolejšová and Denisa Kera. 2017. Soylent diet self-experimentation: Design challenges in extreme citizen science projects. In Proceedings of the 2017 ACM conference on computer supported cooperative work and social computing (CSCW '17), 2112-2123. https://doi.org/10.1145/2998181.2998365

16. Markéta Dolejšová, Danielle Wilde, Ferran Altarriba Bertran, and Hilary Davis. 2020. Disrupting (More-than-) Human-Food Interaction: Experimental Design, Tangibles and Food-Tech Futures. In Proceedings of the 2020 ACM on Designing Interactive Systems Conference (DIS '20), 993-1004. https://doi.org/10.1145/3357236.3395437

17. Jennifer Fereday and Eimear Muir-Cochrane. 2006. Demonstrating Rigor Using Thematic Analysis: A Hybrid Approach of Inductive and Deductive Coding and Theme Development. International Journal of Qualitative Methods 5, 1: 80-92. https://doi.org/10.1177/160940690600500107

18. Bill Gaver, Tony Dunne, and Elena Pacenti. 1999. Design: Cultural Probes. interactions 6, 1: 21-29. https://doi.org/10.1145/291224.291235

19. William W. Gaver, Andrew Boucher, Sarah Pennington, and Brendan Walker. 2004. Cultural Probes and the Value of Uncertainty. interactions 11, 5: 53-56. https://doi.org/10.1145/1015530.1015555

20. Tom Gayler. 2017. Towards edible interfaces: Designing interactions with food. In Proceedings of the 19th ACM international conference on multimodal interaction (ICMI '17), 623-627. https://doi.org/10.1145/3136755.3137030

21. Tom Gayler and Corina Sas. 2017. An Exploration of Taste-emotion Mappings from the Perspective of Food Design Practitioners. In Proceedings of the 2Nd ACM SIGCHI International Workshop on Multisensory Approaches to Human-Food Interaction (MHFI 2017), 23-28. https://doi.org/10.1145/3141788.3141793

22. Tom Gayler, Corina Sas, and Vaiva Kalnikaite. 2019. Taste Your Emotions: An Exploration of the Relationship Between Taste and Emotional Experience for HCI. In Proceedings of the 2019 on Designing Interactive Systems Conference (DIS '19), 1279-1291. https://doi.org/10.1145/3322276.3322336

23. Tom Gayler, Corina Sas, and Vaiva Kalnikaite. 2020. Material Food Probe: Personalized 3D Printed Flavors for Emotional Communication in Intimate Relationships. In Proceedings of the 2020 ACM on Designing Interactive Systems Conference (DIS '20), 965-978. https://doi.org/10.1145/3357236.3395533

24. Tom Gayler, Corina Sas, and Vaiva Kalnikaite. 2021. Framing the Design Space for Food-Human-Technology Interaction. Under revision for ToCHI. 25. Connor Graham, Mark Rouncefield, Martin Gibbs, Frank Vetere, and Keith Cheverst. 2007. How Probes Work. In Proceedings of the 19th Australasian Conference on Computer-Human Interaction: Entertaining User Interfaces (OZCHI '07), 29-37. https://doi.org/10.1145/1324892.1324899

26. Seo Yoon Han and Eun Ji Kang. 2017. ChilDish: The Smart Plate and Cup for Children. In Proceedings of the Companion of the 2017 ACM/IEEE International Conference on Human-Robot Interaction (HRI '17), 391-392. https://doi.org/10.1145/3029798.3034946

27. Adam W. Hanley, Wolf E. Mehling, and Eric L. Garland. 2017. Holding the body in mind: Interoceptive awareness, dispositional mindfulness and psychological well-being. Journal of Psychosomatic Research 99: 13-20. https://doi.org/10.1016/j.jpsychores.2017.05.014

28. Kristina Höök. 2018. Designing with the Body: Somaesthetic Interaction Design. MIT Press.

29. Youjin Hwang, Siyoung Lee, Hyeong Seok Jeon, Jung Han Yoon Park, Ki Won Lee, and Joonhwan Lee. 2018. "Eat What You Want and Be Healthy!": Comfort food effects: Human-food interaction in view of celebratory technology. In Proceedings of the 3rd international workshop on multisensory approaches to human-food interaction (MHFI'18). https://doi.org/10.1145/3279954.3279958 
30. Katherine Isbister, Kristina Höök, Michael Sharp, and Jarmo Laaksolahti. 2006. The Sensual Evaluation Instrument: Developing an Affective Evaluation Tool. In Proceedings of the SIGCHI Conference on Human Factors in Computing Systems (CHI '06), 1163-1172. https://doi.org/10.1145/1124772.1124946

31. Azusa Kadomura, Cheng-Yuan Li, Koji Tsukada, Hao-Hua Chu, and Itiro Siio. 2014. Persuasive technology to improve eating behavior using a sensorembedded fork. In Proceedings of the 2014 ACM international joint conference on pervasive and ubiquitous computing (UbiComp '14), 319-329. https://doi.org/10.1145/2632048.2632093

32. Silke Manuela Kärcher, Sandra Fenzlaff, Daniela Hartmann, Saskia Kathi Nagel, and Peter König. 2012. Sensory Augmentation for the Blind. Frontiers in Human Neuroscience 6. https://doi.org/10.3389/fnhum.2012.00037

33. Rohit Ashok Khot, Deepti Aggarwal, Ryan Pennings, Larissa Hjorth, and Florian "Floyd" Mueller. 2017. EdiPulse: Investigating a playful approach to self-monitoring through 3D printed chocolate treats. In Proceedings of the 2017 CHI conference on human factors in computing systems (CHI '17), 65936607. https://doi.org/10.1145/3025453.3025980

34. Naoya Koizumi, Hidekazu Tanaka, Yuji Uema, and Masahiko Inami. 2011. Chewing jockey: Augmented food texture by using sound based on the cross-modal effect. In Proceedings of the 8th international conference on advances in computer entertainment technology (ACE '11). https://doi.org/10.1145/2071423.2071449

35. Dannie Korsgaard, Thomas Bjørner, Pernille Krog Sørensen, and Jon Ram Bruun-Pedersen. 2019. Older adults eating together in a virtual living room: Opportunities and limitations of eating in augmented virtuality. In Proceedings of the 31st european conference on cognitive ergonomics (ECCE 2019), 168-176. https://doi.org/10.1145/3335082.3335093

36. Stacey Kuznetsov, Christina J. Santana, and Elenore Long. 2016. Everyday food science as a design space for community literacy and habitual sustainable practice. In Proceedings of the 2016 CHI conference on human factors in computing systems (CHI '16), $1786-1797$. https://doi.org/10.1145/2858036.2858363

37. Yujin Lee, Jee Bin Yim, Daye Kang, HyeonBeom Yi, and Daniel Sakes. 2019. Designing internal structure of chocolate and its effect on food texture. In Companion publication of the 2019 on designing interactive systems conference 2019 companion (DIS '19 companion), $231-235$. https://doi.org/10.1145/3301019.3323896

38. Jin-Ling Lo, Tung-Yun Lin, Hao-Hua Chu, Hsi-Chin Chou, Jen-Hao Chen, Jane Yung-Jen Hsu, and Polly Huang. 2007. Playful tray: Adopting ubicomp and persuasive techniques into play-based occupational therapy for reducing poor eating behavior in young children. In Proceedings of the 9th international conference on ubiquitous computing (UbiComp '07), 38-55.

39. Anna Luusua, Johanna Ylipulli, Marko Jurmu, Henrika Pihlajaniemi, Piia Markkanen, and Timo Ojala. 2015. Evaluation Probes. In Proceedings of the 33rd Annual ACM Conference on Human Factors in Computing Systems - CHI '15, 85-94. https://doi.org/10.1145/2702123.2702466

40. Kate McLean. Sensory Maps. Sensory Maps. Retrieved April 22, 2021 from https://sensorymaps.com/

41. Morten C. Meilgaard, B. Thomas Carr, and Gail Vance Civille. 2006. Sensory Evaluation Techniques, Fourth Edition. CRC Press.

42. Sevi Merter. 2017. Synesthetic Approach in the Design Process for Enhanced Creativity and Multisensory Experiences. The Design Journal 20, sup1: S4519-S4528. https://doi.org/10.1080/14606925.2017.1352948

43. Bruno Mesz, Kevin Herzog, Juan Cruz Amusategui, Lucas Samaruga, and Sebastián Tedesco. 2017. Let's drink this song together: Interactive tastesound systems. In Proceedings of the 2nd ACM SIGCHI international workshop on multisensory approaches to human-food interaction (MHFI 2017), 1317. https://doi.org/10.1145/3141788.3141791

44. Christiane Moser and Manfred Tscheligi. 2013. Playful Taste Interaction. In Proceedings of the 12th International Conference on Interaction Design and Children (IDC '13), 340-343. https://doi.org/10.1145/2485760.2485828

45. Florian "Floyd" Mueller, Josh Andres, Joe Marshall, Dag Svan la es, m. c. schraefel, Kathrin Gerling, Jakob Tholander, Anna Lisa Martin-Niedecken, Elena Márquez Segura, Elise van den Hoven, Nicholas Graham, Kristina Höök, and Corina Sas. 2018. Body-centric Computing: Results from a Weeklong Dagstuhl Seminar in a German Castle. Interactions 25, 4: 34-39. https://doi.org/10.1145/3215854

46. Marianna Obrist, Rob Comber, Sriram Subramanian, Betina Piqueras-Fiszman, Carlos Velasco, and Charles Spence. 2014. Temporal, affective, and embodied characteristics of taste experiences: A framework for design. In Proceedings of the SIGCHI conference on human factors in computing systems (CHI '14), 2853-2862. https://doi.org/10.1145/2556288.2557007

47. Marianna Obrist, Yunwen Tu, Lining Yao, and Carlos Velasco. 2019. Space Food Experiences: Designing Passenger's Eating Experiences for Future Space Travel Scenarios. Frontiers in Computer Science 1. https://doi.org/10.3389/fcomp.2019.00003

48. Marianna Obrist, Carlos Velasco, Chi Vi, Nimesha Ranasinghe, Ali Israr, Adrian Cheok, Charles Spence, and Ponnampalam Gopalakrishnakone. 2016. Sensing the future of HCI: touch, taste, and smell user interfaces. Interactions 23, 5: 40-49. https://doi.org/10.1145/2973568

49. Gaurav Patekar, Karan Dudeja, Himanshu Bablani, and Debanshu Bhaumik. 2018. Data Jalebi Bot. In Extended Abstracts of the 2018 CHI Conference on Human Factors in Computing Systems (CHI EA '18), 1-4. https://doi.org/10.1145/3170427.3186528

50. Sarah Pink, Kerstin Leder Mackley, Val Mitchell, Marcus Hanratty, Carolina Escobar-Tello, Tracy Bhamra, and Roxana Morosanu. 2013. Applying the lens of sensory ethnography to sustainable HCI. ACM Transactions on Computer-Human Interaction 20, 4: 25:1-25:18. https://doi.org/10.1145/2494261

51. Mirjana Prpa, Sarah Fdili-Alaoui, Thecla Schiphorst, and Philippe Pasquier. 2020. Articulating Experience: Reflections from Experts Applying MicroPhenomenology to Design Research in HCI. In Proceedings of the 2020 CHI Conference on Human Factors in Computing Systems (CHI '20), 1-14. https://doi.org/10.1145/3313831.3376664

52. Stephen Rayner and Richard Riding. 1997. Towards a Categorisation of Cognitive Styles and Learning Styles. Educational Psychology 17, 1-2: 5-27. https://doi.org/10.1080/0144341970170101

53. Joanne Riekhoff and Panos Markopoulos. 2008. Sampling young children's experiences with cultural probes. In Proceedings of the 7th international conference on Interaction design and children (IDC '08), 145-148. https://doi.org/10.1145/1463689.1463742

54. Corina Sas, Kobi Hartley, and Muhammad Umair. 2020. ManneqKit Cards: A Kinesthetic Empathic Design Tool Communicating Depression Experiences. In Proceedings of the 2020 ACM Designing Interactive Systems Conference (DIS '20), 1479-1493. https://doi.org/10.1145/3357236.3395556 55. Corina Sas and Chenyan Zhang. 2010. Do emotions matter in creative design? In Proceedings of the 8th ACM Conference on Designing Interactive Systems, 372-375.

56. Hendrik N. J. Schifferstein. 2011. Multi sensory design. In Procedings of the Second Conference on Creativity and Innovation in Design (DESIRE '11), 361-362. https://doi.org/10.1145/2079216.2079270

57. Thecla Schiphorst, Lian Loke, and Kristina Höök. 2020. Designing for Sensory Appreciation: Cultivating Somatic Approaches to Experience Design. In Extended Abstracts of the 2020 CHI Conference on Human Factors in Computing Systems (CHI EA '20), 1-4. https://doi.org/10.1145/3334480.3375056 58. Dag Svanæs and Louise Barkhuus. 2020. The Designer's Body as Resource in Design: Exploring Combinations of Point-of-view and Tense. In Proceedings of the 2020 CHI Conference on Human Factors in Computing Systems, 1-13. 
59. Laia Turmo Vidal, Elena Márquez Segura, and Annika Waern. 2018. Sensory bodystorming for collocated physical training design. In Proceedings of the 10th Nordic Conference on Human-Computer Interaction (NordiCHI '18), 247-259. https://doi.org/10.1145/3240167.3240224

60. Paula Varela and Gastón Ares. 2012. Sensory profiling, the blurred line between sensory and consumer science. A review of novel methods for product characterization. Food Research International 48, 2: 893-908. https://doi.org/10.1016/j.foodres.2012.06.037

61. Chi Thanh Vi, Daniel Arthur, and Marianna Obrist. 2018. TasteBud: Bring taste back into the game. In Proceedings of the 3rd international workshop on multisensory approaches to human-food interaction (MHFI'18). https://doi.org/10.1145/3279954.3279955

62. Jayne Wallace, John McCarthy, Peter C. Wright, and Patrick Olivier. 2013. Making Design Probes Work. In Proceedings of the SIGCHI Conference on Human Factors in Computing Systems (CHI '13), 3441-3450. https://doi.org/10.1145/2470654.2466473

63. Beverley Merle Watts, G. L. Ylimaki, L. E. Jeffery, and Luiz G. Elias. 1989. Basic sensory methods for food evaluation. IDRC, Ottawa, ON, CA.

Retrieved from https://idl-bnc-idrc.dspacedirect.org/bitstream/handle/10625/2844/IDL-2844.pdf?sequence=1

64. Jun Wei, Adrian David Cheok, and Ryohei Nakatsu. 2012. Let's have dinner together: Evaluate the mediated co-dining experience. In Proceedings of the 14th ACM international conference on multimodal interaction (ICMI '12), 225-228. https://doi.org/10.1145/2388676.2388721

65. Danielle Wilde, Anna Vallgårda, and Oscar Tomico. 2017. Embodied Design Ideation Methods: Analysing the Power of Estrangement. In Proceedings of the 2017 CHI Conference on Human Factors in Computing Systems - CHI '17, 5158-5170. https://doi.org/10.1145/3025453.3025873

66. GUESS WHO | Hasbro Games. Retrieved July 7, 2020 from https://products.hasbro.com/en-gb/product/guess-who:2CE41484-19B9-F369-D94AA92637F6C809 